\title{
Electron Tomography of Gold Nanoparticles in Titania Composite Aerogels: Probing Structure to Understand Photochemistry
}

\author{
Todd H. Brintlinger ${ }^{1}$, Paul A. DeSario ${ }^{2}$, Jeremy J. Pietron ${ }^{2}$, Lindsey C. Szymczak ${ }^{2}$, Rhonda M. Stroud ${ }^{1}$, \\ and Debra R. Rolison ${ }^{2}$ \\ 1. Materials Science and Technology Division, U.S. Naval Research Laboratory, Washington, DC, USA \\ 2. Chemistry Division, U.S. Naval Research Laboratory, Washington, DC, USA 20375
}

High surface area titania aerogels show potential as photocatalysts for a range of applications, from solar energy conversion ${ }^{1}$ to environmental remediation ${ }^{2}$. However, a large amount of the solar spectrum falls outside the energetic wavelengths needed to drive the photochemistry associated with a large bandgap semiconductor such as titania. Development of titania-based materials that are active in the visible range of the spectrum could significantly improve the photocatalytic performance and extend the range of applications. One approach is to incorporate metal nanoparticles to induce a lower energy absorption, through photon interaction with local surface plasmon resonances on metal nanoparticles. The effects of the metal particle size, shape, and distribution on the photocatatyic activity have been well documented for variety of semiconductors ${ }^{1}$. We find here that the positional relationship between the metals and their semiconducting scaffolding may be similarly significant.

We previously demonstrated that composite metal-loaded aerogels ${ }^{2}$, in which metal nanoparticles are added just as the matrix material begins to gel, show enhanced photocatalytic activity over 'conventional' metal-loaded aerogels in which the metals are added after aerogel formation. Photooxidation of methanol measurements indicate the difference in photocatalytic activity is due to a difference in the structural relationship between gold particles and titania aerogel mesoparticle network. In order to provide a direct and quantitative confirmation of the difference in gold-titania arrangement and relationship to photocatalytic activity, we characterize these samples with transmission electron microscopy.

First, to rule out effects resulting from metal nanoparticle size, shape, and distribution, we imaged the conventional and composite aerogels using STEM-HAADF, as seen in Fig. 1. Following particle analysis that involved both automated thresholding and manual identification, aerogels show gold nanoparticles with mean $4.7 \mathrm{~nm}$ diameter, with 0.4 and $0.5 \mathrm{~nm}$ standard deviation for conventional and composite aerogel synthesis, respectively. Eccentricities show similarly narrow distributions. However, these analyses were performed on just a single imaging plane (at $0^{\circ}$ tilt).

Electron tomograms of samples with gold incorporated during and after titania gelation were acquired. Bright field TEM image tilt series of each sample were obtained with a JEOL 2202FS operated at 200kV using a Fishcione (Model 2030) high-tilt tomography holder. Individual micrographs were taken with $300 \mathrm{~nm}$ underfocus using serialEM control software ${ }^{4}$ using $2^{\circ}$ tilt increments, with a typical full tilt range of $+68^{\circ}$ to $-68^{\circ}$. Tomograms were assembled using etomo software package ${ }^{5}$ in which the embedded gold nanoparticles were used as fiducials and manually tracked. Compiled tomograms then allowed for segmentation of gold nanoparticles from titania, as well as removal of holey carbon support, within Avizo Fire. ${ }^{6}$ Finally, a series of binary morphological operations were performed using custom Matlab codes to reduce noise before final analysis and visualization. Results of these analyses are seen in Fig. 2. For comparison of connectivity between gold and titania, a binary distance operation was 
performed on the segmented gold nanoparticle datacube such that neighboring titania mesoparticles could be identified. Following this, a 3-d watershed allowed for separate analysis of selections of titania associated with a given gold particle. Here, the volume of titania within $<1 \mathrm{~nm}$ of each gold nanoparticle is larger for the composite aerogels, indicating larger gold-titania contact interface area. Our results confirm that increased connectivity between the gold nanoparticles and the titania mesoporous network in composite aerogels enhances the photocatalytic activity. Additional research is underway to determine the specific mechanism for the enhancement, e.g. higher surface energies at an extended gold-titania interface or increased gas transport.

\section{References:}

[1] S. Linic, P. Christopher and D.B. Ingram, Nat. Mater 10, p. 911 (2011).

[2] T. L. Thompson and J. T. Yates, Jr., Chem. Rev. 106, p. 4428 (2006).

[3] J. J. Pietron, R. M. Stroud and D.R. Rolison, Nano Lett. 2, p. 545 (2002).

[4] D.N. Mastronarde, J. Struct. Biol. 152, p.36 (2005) and http://bio3d.colorado.edu/SerialEM/.

[5] D.N. Mastronarde, J. Struct. Biol. 120, p. 343 (1997) and http://bio3d.colorado.edu/imod/.

[6] www.vsg3d.com/avizo/fire
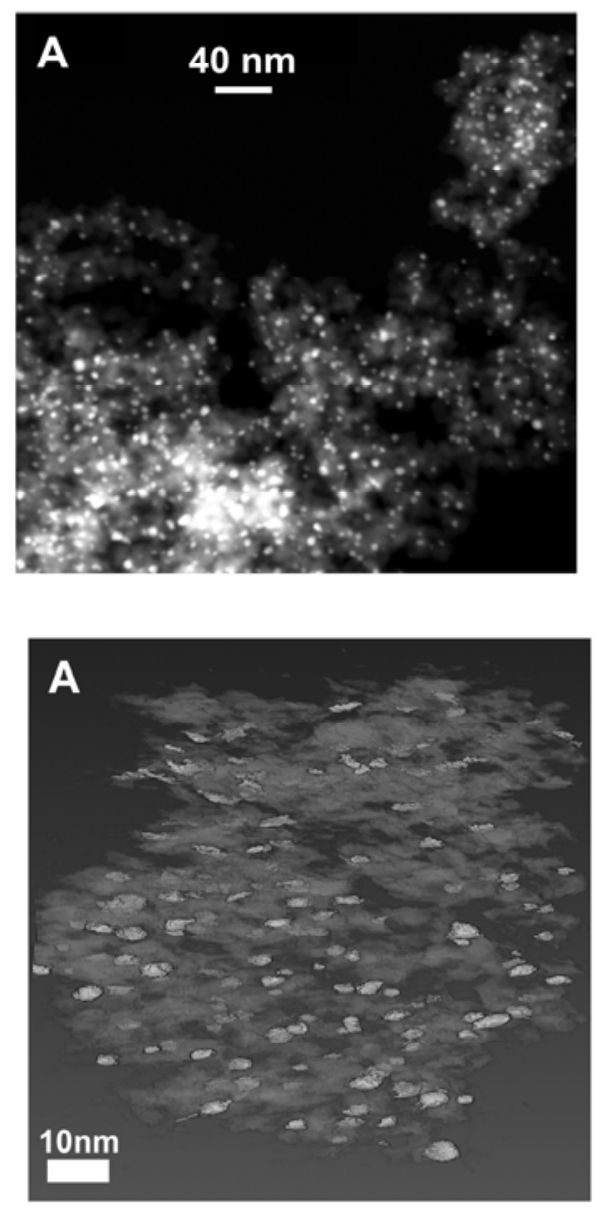
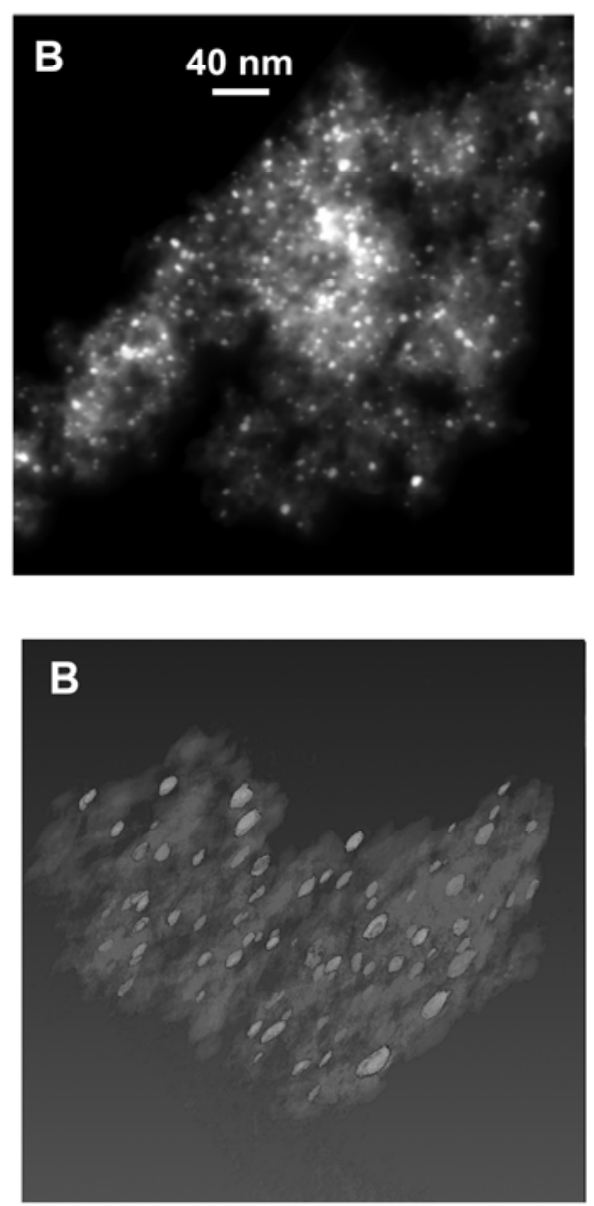

Fig. 1. STEM-HAADF micrographs comparing shape, size, and distribution of gold nanoparticles and titania aerogel. A) Aerogel with gold particles deposited after aerogel synthesis. B) Aerogel with gold nanoparticles grown during aerogel synthesis.

Fig. 2. Electron tomograms of titania aerogels and gold nanoparticles. A) Conventional aerogel with gold particles deposited after aerogel synthesis. Gold particles are lighter color. B) Composite aerogel with gold nanoparticles grown during aerogel synthesis. Scales are same for both images. 